\title{
Benefícios da farinha do maracujá amarelo (Passiflora Edulis F. Flavicarpa Deg.) no tratamento do Diabetes mellitus tipo 2: Uma revisão narrativa
}

\author{
Benefits of the flour of the yellow passion fruit (Passiflora Edulis F. Flavicarpa Deg.) in the \\ treatment of Diabetes mellitus type 2: A narrative review \\ Beneficios de la harina de maracuyá amarilla (Passiflora Edulis F. Flavicarpa Deg.) en el \\ tratamiento de la Diabetes mellitus tipo 2: Una revisión narrativa
}

Recebido: 19/07/2021 | Revisado: 25/07/2021 | Aceito: 26/07/2021 | Publicado: 01/08/2021

Raíssa de Oliveira Balthar
ORCID: https://orcid.org/0000-0002-0983-3119
E-mail: raissa.balthar@ gmail.com
Universidade Federal do Estado do Rio de Janeiro, Brasil
Anna Paula Oliveira Alvarez Maciel
ORCID: https://orcid.org/0000-0002-6094-341X
E-mail: anna.oliveira@edu.unirio.br
Célia Cristina Diogo Ferreira
Universidade Federal do Esto do Rio de aneiro, Brasil
ORCID: https://orcid.org/0000-0002-9561-3277
Universidade Federal do Rio de Janeiro, Brasil
E-mail: celiacdf@gmail.com

\section{Resumo}

Introdução: O Diabetes Mellitus tipo 2 é uma síndrome caracterizada por hiperglicemia, resultante de defeitos na secreção da insulina, ação da insulina ou ambas, em tecidos-alvos (fígado, músculo e tecido adiposo). O presente estudo teve como objetivo verificar os efeitos terapêuticos da suplementação da farinha da casca do maracujáamarelo (Passiflora Edulis f. flavicarpa Deg.) no tratamento de indivíduos acometidos por Diabetes Mellitus tipo 2. Metodologia: Trata-se de uma revisão narrativa, realizada por meio da seleção e análise de artigos científicos e trabalhos acadêmicos publicados entre os anos 2011 e 2021. Desenvolvimento: A partir do levantamento dos estudos, identificou-se que a farinha da casca do maracujá amarelo, apresenta efeitos benéficos principalmente no controle glicêmico, devido à presença de fibras solúveis, como a pectina, presente em sua casca, que aumenta o teor de fibras da dieta, reduzindo assim, a glicemia e os lipídeos circulantes no indivíduo diabético. Conclusão: As informações trazidas por este trabalho sugerem o uso da farinha de casca de maracujá como terapia complementar dos tratamentos convencionais e podem auxiliar os profissionais de saúde na implementação de estratégias que auxiliem os pacientes diabéticos em escolhas alimentares mais saudáveis.

Palavras-chave: Passiflora; Suplementos Nutricionais; Doenças Metabólicas; Pectina.

\begin{abstract}
Introduction: Type 2 Diabetes Mellitus is a syndrome characterized by hyperglycemia, resulting from defects in insulin secretion, insulin action or both, in target tissues (liver, muscle and adipose tissue). This study aimed to verify the therapeutic effects of supplementation of yellow passion fruit peel flour (Passiflora Edulis f. flavicarpa Deg.) in the treatment of individuals affected by type 2 Diabetes Mellitus. Methodology: This is a narrative review, carried out through the selection and analysis of scientific articles and academic papers published between 2011 and 2021. Development: From the survey of studies, it was identified that yellow passion fruit peel flour has beneficial effects mainly in the control glycemic, due to the presence of soluble fibers, such as pectin, present in its bark,
\end{abstract}


which increases the fiber content of the diet, thus reducing blood glucose and circulating lipids in the diabetic individual. Conclusion: The information provided by this study suggests the use of passion fruit peel flour as a complementary therapy to conventional treatments and can help health professionals to implement strategies that help diabetic patients make healthier food choices.

Keywords: Passionflower; Nutritional Supplements; Metabolic Diseases; Pectin.

\section{Resumen}

Introducción: La diabetes mellitus tipo 2 es un síndrome caracterizado por hiperglucemia, resultante de defectos en la secreción de insulina, la acción de la insulina, o ambas, en los tejidos diana (hígado, músculo y tejido adiposo). Este estudio tuvo como objetivo verificar los efectos terapéuticos de la suplementación con harina de cáscara de maracuyá amarilla (Passiflora edulis f. Flavicarpa Deg.) En el tratamiento de individuos afectados por Diabetes Mellitus tipo 2. Metodología: Se trata de una revisión narrativa, realizada por a través de la selección y análisis de artículos científicos y trabajos académicos publicados entre 2011 y 2021. Desarrollo: A partir del relevamiento de estudios, se identificó que la harina de cáscara de maracuyá amarilla tiene efectos beneficiosos principalmente en el control glucémico, debido a la presencia de fibras solubles, como la pectina, presentes en su corteza, que aumenta el contenido de fibra de la dieta, reduciendo así la glucosa en sangre y los lípidos circulantes en el individuo diabético. Conclusión: La información que aporta este trabajo sugiere el uso de la harina de cáscara de maracuyá como terapia complementaria a los tratamientos convencionales y puede ayudar a los profesionales de la salud en la implementación de estrategias que ayuden a los pacientes diabéticos en opciones de alimentos más salud.

Palabras clave: Pasiflora; Complementos Nutricionales; Enfermedades Metabólicas; Pectina.

\section{Introdução}

O Diabetes Mellitus (DM) é considerado um problema de saúde pública em vários países do mundo, devido à alta prevalência na população, complicações crônicas, mortalidade, altos custos financeiros e sociais referentes ao tratamento e perda na qualidade de vida (BRASIL, 2013). O DM é uma enfermidade que não escolhe nível social, etnia, gênero, cultura e principalmente idade. Logo, pode haver um aumento significativo devido aos fatores de risco que essa doença apresenta, como: obesidade, sedentarismo, alimentação inadequada, histórico familiar, envelhecimento populacional e estar associada a outras enfermidades (Petermann et al., 2018; Rojas-Padilla, Zambrano-Ríos \& Matta- Miramar, 2020).

O DM tipo 2 (DM2) pode ser caracterizado por uma combinação de resistência à insulina e por falha da célula $\beta$, em que os níveis endógenos de insulina podem estar normais, reduzidos ou aumentados, contudo, encontram-se inadequados para superar a resistência à insulina concomitante (redução na sensibilidade tecidual ou capacidade de resposta à insulina). Como resultado, ocorre a hiperglicemia (Franz \& Evert, 2018), que quando mal controlada, está associada às complicações, disfunções e insuficiência de órgãos, como olhos, rins, cérebro, coração, entre outros (Dias da Silva et al., 2020)

A preocupação com indivíduos com DM torna-se mais evidente no atual momento, uma vez que está entre as comorbidades mais frequentes relatas em pacientes infectados com COVID-19, visto que este prejudica o equilíbrio glicêmico e favorece a ocorrência de complicações metabólicas, como a cetoacidose. Com isso, nota-se que indivíduos com DM estão mais propensos a desenvolverem a forma mais grave e crítica da doença do que indivíduos saudáveis, uma vez que diabéticos necessitam de internação em terapia intensiva e/ou uso de ventilação mecânica invasiva (VMI), sendo assim, considerados do grupo de risco (Oriolli et al., 2020). Até a primeira quinzena de julho, no Brasil, já havido sido registrados 533.488 óbitos, sendo 58.808 no estado do Rio de Janeiro (Brasil, 2021). 
Dentre os cuidados dietoterápicos com os pacientes diabéticos, estão a administração do tipo e a quantidade de carboidrato a ser consumido, uma vez que no cardápio destes indivíduos deve-se dar preferência aos carboidratos complexos. Este nutriente, como as fibras alimentares são absorvidos mais lentamente no organismo, e estão intimamente relacionadas com as melhoras no perfil lipídico, na pressão arterial e na sensibilidade à insulina (Cunha, Campos \& Silva, 2017).

A farinha do maracujá amarelo (Passiflora edulis f. flavicarpa Deg.) é rica em pectina, uma fibra solúvel encontrada na entrecasca do maracujá e que promove retardo do esvaziamento gástrico, aumenta o poder de saciedade, retarda o tempo de absorção dos carboidratos simples, ajuda a normalizar a glicose sanguínea e os níveis de insulina, bem como complexa os sais biliares e o colesterol, aumentando sua excreção (Oliveira, 2016; Santana et al., 2011).

Estudos comprovaram que a administração de $30 \mathrm{~g}$ por dia da farinha da casca de Passiflora edulis é segura para consumo, visto que essa quantidade consumida não apresentou alterações que indicassem sinais de toxicidade nos exames físicos e laboratoriais que pudessem implicar no seu uso como alimento funcional. Além disso, demonstraram a ação benéfica no controle da glicemia, atuando como auxiliar nas terapias em pacientes com DM2, tendo assim, efeito favorável sobre a sensibilidade à insulina (Araújo et al., 2018; Cunha, 2018; Gaspar, Araújo \& Uchoa, 2016; Queiroz et al., 2012).

Por ser uma fruta altamente perecível, a transformação do maracujá em farinha pode ser uma forma de melhor aproveitá-la e garantir uma vida de prateleira ainda maior, podendo ser incorporada em produtos alimentícios (Ozores, Storck \& Fogaça, 2015).

Neste contexto, procurando aumentar o conhecimento sobre os benefícios da farinha do maracujá amarelo, o objetivo deste trabalho foi abordar por meio de uma revisão narrativa da literatura, o efeito do consumo da farinha do maracujá amarelo (Passiflora edulis f. flavicarpa Deg.) no tratamento do DM2.

\section{Metodologia}

Esta revisão narrativa da literatura foi realizada em bancos de dados eletrônicos nos principais periódicos científicos, com a utilização das bases de dados Embase, Periódicos Capes, Pubmed, Science Direct, Scientific Eletronic Library Online (Scielo), Scopus e Wef of Science, assim como manuais e sites do Ministério da Saúde no período de 2011-2021, a fim de abordar informações obtidas nos textos científicos encontrados nessas bases eletrônicas. Para a elaboração deste texto foram seguidas as normas metodológicas descritas por Elias et al. (2012). Para isso, foram utilizadas as palavras-chaves, em inglês e português, isolados e combinados: Passiflora edulis, Suplementos Nutricionais, Doenças Metabólicas e Pectina. Os trabalhos foram limitados à língua portuguesa, inglesa e espanhola.

Inicialmente foram considerados os títulos e os resumos dos artigos para a seleção ampla de prováveis trabalhos de interesse, sendo destacados os resumos (dos artigos que não tinham texto acessível) e os textos completos dos artigos. Foram critérios de exclusão: artigos em outros idiomas que não os selecionados; artigos que não se encaixem no período selecionado, trabalhos científicos realizados com animais, trabalhos realizados com gestante, trabalhos relacionados à obesidade, composição físico-química da farinha de maracujá e de produtos desenvolvidos com esta farinha.

\section{Desenvolvimento}

\section{Diabetes Mellitus- epidemiologia e classificação}

Nas últimas décadas a população brasileira passou por diversas mudanças como a transição demográfica, em que houve um aumento da expectativa de vida da população, com uma proporção muito maior da população idosa; transição epidemiológica, com a diminuição das doenças infecciosas e o aumento das doenças crônicas e a transição nutricional. Nesta última, o perfil nutricional do brasileiro sofreu modificações, passando de estado de desnutrição para o de sobrepeso e obesidade, fato que está intimamente relacionado com o aumento significativo no consumo de alimentos industrializados e alta 
densidade energética, que por serem mais acessíveis e instantâneos oferecem maior facilidade e comodidade ao consumidor (Brasil, 2013; Franz \& Evert, 2018; Sociedade Brasileira de Diabetes [SBD], 2019).

Em pleno século XXI, a alimentação da população já não é mais a mesma, se comparada com a do século passado, o que acarretou o aumento da prevalência da obesidade, consequência de maus hábitos alimentares e um estilo de vida sedentário, estando assim, intimamente ligadas ao desenvolvimento de Doenças Crônicas Não - Transmissíveis (DCNTs), como por exemplo, o DM2 (Kahn, Cooper \& Del Prato, 2014).

De acordo com a SBD (2019), DM é uma doença crônica e metabólica, caracterizada por hiperglicemia, que pode ser resultante de defeitos na secreção/ação de insulina ou até mesmo de ambos e quando associadas a complicações, disfunções e insuficiência de vários órgãos como os olhos, rins, nervos, cérebro, coração e vasos sanguíneos (International Diabetes Federation [IDF], 2017). Com a mudança nos padrões alimentares, pode-se observar o avanço do número de indivíduos diagnosticados com DM2. As estimativas apontam que no Brasil já são mais de 16,8 milhões de indivíduos acometidos pela DM2 (IDF, 2019), sendo a maior prevalência em indivíduos apresentando 65 anos ou mais e do sexo masculino (Brasil, 2018). O DM pode ser classificado em DM tipo 1 (DM1), que pode subdividir-se em imunomediada ou idiopática, compreendendo 5 a $10 \%$ do total de casos; DM2, que compreende cerca de 90 a 95\% do total de casos; outros tipos específicos de diabetes e a DM gestacional (SBD, 2019).

\section{Fisiopatologia do Diabetes Mellitus tipo 2}

O DM2 é causado por defeitos na ação da insulina, na excreção de insulina ou em ambos os casos, impedindo a entrada da glicose nas células para sua metabolização provocando glicotoxicidade, que caracteriza-se por efeitos adversos da hiperglicemia crônica sobre a função da célula beta e incluem três consequências distintas: diminuição da tolerância à glicose; exaustão das células beta e redução da massa de células beta por apoptose. A produção da insulina ocorre nas ilhotas de Langerhans no pâncreas, o órgão mais importante para a síntese da insulina endógena crônicas do diabetes (Forcina, Almeida \& Ribeiro-Jr., 2008; Franz \& Evert, 2018; SBD, 2019).

Os efeitos insulínicos são exercidos por meio de receptores que são ativados pela insulina: fator de crescimento semelhante à insulina tipo 1 e tipo 2 (IGF-I, IGF-II), que estão presentes nas superfícies dos leucócitos. Há receptores no fígado, músculo e gordura, que são sensíveis à insulina, tendo assim, diversos efeitos sobre o metabolismo energético desses tecidos, e o maior deles é o de regulação da glicose, armazenamento de glicogênio, armazenamento de ácidos graxos e síntese de proteína (Talia, Connolly \& Fowler, 2021).

\section{Relação entre DM e COVID-19}

A pandemia da COVID-19 é causada pela síndrome respiratória aguda grave (SARS - Cov - 2). A transmissão dessa doença ocorre de maneira direta, ou seja, por contato e por aerossol de gotículas respiratórias e tem um tempo médio de incubação de cinco (5) dias. Apresenta como sintomas mais comuns: febre, tosse, dispneia (falta de ar) e mialgias (dor muscular) ou fadigas (Zanaib Shahib et al. 2020).

Indivíduos com doenças crônicas já possuem um processo inflamatório crônico e lesão em vários órgãos, com isso, pode-se encontrar na literatura a ocorrência de pior prognóstico em doenças virais naqueles indivíduos que apresentam comorbidades como: Hipertensão arterial, DM, Obesidade, Síndrome Metabólica (SM) e outras DCNT's (DIAS et al., 2020).

Um estudo realizado por Zhu et al. (2020) demonstrou que a DM é fator de risco para a progressão da COVID-19 e seus desfechos. Além disso, a glicose sanguínea quando controlada, está associada à redução significativa tanto dos resultados adversos quanto da morte. Corroborando com esse estudo, Zhang et al. (2020) estudaram pacientes com COVID-19 e DM e notaram que esses indivíduos apresentavam maior probabilidade de desenvolver doenças graves ou críticas com mais 
complicações e apresentaram maiores taxas de incidência de antibioticoterapia, ventilação mecânica não invasiva, invasiva e morte.

Com isso, fica evidente que indivíduos diabéticos devam receber, de acordo com as diretrizes da SBD (2019), um atendimento individualizado, ou seja, cada indivíduo deve receber uma recomendação específica quanto à terapia nutricional, bem como possuir um plano alimentar para um processo educativo contínuo. Sabendo-se que a terapia nutricional do indivíduo diabético deve-se concentrar no equilíbrio dos macronutrientes para a manutenção do bom controle metabólico, faz-se necessário a presença do nutricionista para implementar as intervenções e a educação nutricional para estes indivíduos.

\section{Propriedades funcionais da farinha de maracujá amarelo no Diabetes Mellitus}

O uso de plantas medicinais está na cultura da população desde a antiguidade e tem sido utilizada para tratamento, cura e prevenção de doenças (Alvarenga, 2017), ganhando espaço no ramo da medicina complementar, já que diversas profissões, dentre elas, a Nutrição, tem adquirido conhecimento de sua aplicação, inserindo a sua utilização nos cuidados da saúde do ser humano (Navolar, Tesser \& Azevedo, 2012). Uma das plantas medicinais que vêm sendo utilizada para auxiliar no tratamento de DCNT, como o DM, destacam-se as espécies de maracujá, dentre elas o maracujá amarelo, também conhecido como Passiflora edulis.

O maracujá amarelo ou maracujá azedo, é a espécie mais produzida e comercializado, representando 95\% dos pomares e seu cultivo está voltado principalmente para a indústria de sucos e polpas, em especial devido ao seu sabor mais ácido e maior rendimento (Cazarin et al., 2014). Sua larga produção está relacionada ao clima e por esta razão, o maracujá é uma fruta tropical nativa do Brasil. Essa fruta é normalmente consumida in natura, da qual utiliza-se sua polpa para a produção de sucos, geleias e sorvetes, acarretando uma grande produção de resíduos ou subprodutos dessa fruta, uma vez que sua casca não é utilizada (Cádiz-Gurrea et al., 2020; Vuolo et al., 2020). Os resíduos do processamento industrial do maracujáamarelo são a casca, o albedo e as sementes, que correspondem a cerca de $40 \%$ a $60 \%$ da massa total da fruta, sendo $12 \%$ a $32 \%$ somente de albedo (Santos, 2017).

A casca de maracujá, composto de flavedo e albedo (parte branca) é constituída por carboidratos, proteínas, é rica em fibras, principalmente fibras solúveis, como pectina. Trata-se de um polímero linear de ácido galacturônico unido por ligações do tipo $\rightarrow$ ( 1 a 4) e é comumente encontrado em casca de frutos em uma concentração de 0,5 a 3,5\% (Lattimer \& Haub, 2010). Há evidências de que na casca de maracujá encontram-se cerca de 2 a 3\% de pectina (Canteri, Wosiacki \& Scheer, 2012). Essa fibra está relacionada indiretamente com o efeito hiperglicêmico, interagindo assim, na digestão do amido, cujo grau de digestão está associado a doenças metabólicas como a obesidade e a diabetes (Muñoz-Almagro, Montilla \& Villamiel, 2021) Além disso, a casca do maracujá apresenta vitaminas e minerais, tais como niacina, ferro, cálcio e fósforo e compostos fenólicos entre outros fitoquímicos, o que contribui para sua utilização na produção e desenvolvimento de alimentos para o consumo humano, já que estes possuem diversas atividades funcionais na prevenção ou amenização de algumas enfermidades (Bruning, Mosegui \& Vianna, 2012; Cádiz-Gurrea et al, 2020).

A farinha do maracujá amarelo é um resíduo da indústria de suco de maracujá (Vuolo et al., 2020), resultante de um processo de moagem de suas cascas desidratadas (Dias et al., 2017). Dessa maneira, diversos estudos tem sido realizados com a farinha de subprodutos de maracujá como ingrediente funcional para o desenvolvimento de diversos tipos de preparações, sendo muito utilizada para elaboração de biscoitos (Lupatini et al., 2011; Miranda, 2014; Santana et al., 2011), bolos (Miranda et al., 2013; Ozores, Storck \& Fogaça, 2015), pães (Ambrósio-Ugri \& Ramos, 2012), barras de cereal (Claro, Rodrigues \& Teixeira, 2018) e macarrão (Costa et al., 2018).

A farinha de maracujá é considerada um alimento funcional devido ao elevado teor de fibras, vitaminas, minerais e compostos bioativos, que possuem diversas atividades funcionais na promoção da saúde (Dias et al., 2017), visto que com o 
aumento da incidência de DCNTs relacionadas à má alimentação, algumas indústrias de alimentos passaram a oferecer produtos capazes de reduzir os riscos de doença e capazes de promover a saúde (Oliveira et al., 2015), melhorando assim, os parâmetros de saúde, como controle da glicemia, dislipidemia e hipertensão em animais e humanos (Vuolo et al., 2020).

Para Claro, Rodrigues \& Teixeira (2018), o uso da farinha da casca do maracujá amarelo na alimentação humana aumenta o teor de fibras da dieta, melhorando o trânsito intestinal de indivíduos constipados, além de contribuir para redução da glicemia e lipídeos circulantes. Segundo Ferreira \& Pena (2010), o elevado teor de fibras alimentares na composição físicoquímica da casca do maracujá amarelo permite que a farinha dela obtida seja considerada um complemento alimentar.

No Quadro 1 pode ser observado que foram selecionados dez artigos de acordo com os critérios estabelecidos na metodologia desta revisão. Os artigos selecionados foram analisados e comparados quanto ao tipo de estudo, local onde foi realizado, amostra utilizada, dose e tempo de uso da farinha da casca de maracujá e resultados encontrados.

Quadro 1. Estudos experimentais com humanos que objetivaram avaliar os efeitos da farinha da casca de maracujá amarelo sobre a glicemia por meio dos marcadores bioquímicos

\begin{tabular}{|c|c|c|c|}
\hline Autor/Ano & Amostra utilizada & População & Principais resultados \\
\hline Castro (2019) & $\begin{array}{l}3 \text { cápsulas contendo } 500 \mathrm{mg} \text { de } \\
\text { farinha de maracujá amarelo (total } \\
4,5 \mathrm{~g} / \mathrm{dia} \text { ), consumidas diariamente } 60 \\
\text { minutos antes das } 3 \text { principais } \\
\text { refeições (desjejum, almoço e jantar), } \\
\text { durante } 120 \text { dias. }\end{array}$ & $\begin{array}{l}56 \text { participantes com DM2, ambos } \\
\text { os sexos, maiores de } 18 \text { anos, } \\
\text { sedentários, obesos }\end{array}$ & $\begin{array}{l}\text { Os resultados demonstraram que houve redução } \\
\text { significativa da glicemia venosa de jejum e a } \\
\text { hemoglobina glicada. Em relação ao controle } \\
\text { lipídico, constatou-se melhora nos níveis de } \\
\text { LDL-C e HDL-C, porém sem significância } \\
\text { estatística. }\end{array}$ \\
\hline Cunha (2018) & $\begin{array}{l}12 \mathrm{~g} / 3 \text { vezes ao dia (antes do } \\
\text { desjejum, almoço e jantar); } \\
56 \text { dias. }\end{array}$ & $\begin{array}{l}54 \text { participantes com DM2, ambos } \\
\text { os sexos, maiores de } 18 \text { anos; } \\
\text { sedentários. }\end{array}$ & $\begin{array}{l}\text { Não houve diferença significativa nos valores de } \\
\text { glicemia capilar, glicemia de jejum e } \\
\text { hemoglobina glicada. }\end{array}$ \\
\hline Silva, (2018) & $\begin{array}{l}60 \text { sachês com } 20 \mathrm{~g} \text { cada. } \\
1 \text { vez/dia no período de } 60 \text { dias }\end{array}$ & $\begin{array}{l}24 \text { indivíduos com DM2 e doença } \\
\text { renal crônica, ambos os sexos, com } \\
\text { média de idade de } 54 \text { anos. }\end{array}$ & $\begin{array}{l}\text { No grupo da farinha notou-se uma diminuição da } \\
\text { glicemia e do colesterol em relação ao grupo } \\
\text { placebo. }\end{array}$ \\
\hline Araújo et al. (2017) & $\begin{array}{l}12 \mathrm{~g} \text { de farinha antes das } 3 \text { principais } \\
\text { refeições(diariamente); } \\
56 \text { dias }\end{array}$ & $\begin{array}{l}54 \text { pessoas com DM2, ambos os } \\
\text { sexos, com idade entre } 18 \text { e } 65 \\
\text { anos. }\end{array}$ & $\begin{array}{l}\text { Pequena redução na porcentagem de } \\
\text { hemoglobina glicada. }\end{array}$ \\
\hline $\begin{array}{l}\text { Gaspar, Araújo \& } \\
\text { Uchoa (2016) }\end{array}$ & $\begin{array}{l}12 \mathrm{~g} \text { de farinha, } 3 \text { vezes ao dia com } \\
\text { um alimento; } \\
56 \text { dias. }\end{array}$ & $\begin{array}{l}54 \text { participantes com DM2, ambos } \\
\text { os sexos, maiores de } 18 \text { anos. }\end{array}$ & $\begin{array}{l}\text { A redução da hemoglobina glicada não foi } \\
\text { estatisticamente significante. }\end{array}$ \\
\hline Costa et al. (2015) & $\begin{array}{l}\text { 50g de massa tipo talharim; } \\
30,60 \text { e } 120 \text { minutos após consumo }\end{array}$ & $\begin{array}{l}20 \text { participantes, não diabéticos, } \\
\text { ambos os sexos, idade entre } 18 \text { e } 59 \\
\text { anos. }\end{array}$ & $\begin{array}{l}\text { Os resultados obtidos confirmam que a adição da } \\
\text { farinha da casca do maracujá em massas } \\
\text { alimentícias contribui para a redução da glicemia. }\end{array}$ \\
\hline Miranda et al. (2014) & $\begin{array}{l}30 \mathrm{~g} \text { de farinha da casca de maracujá; } \\
60 \text { dias }\end{array}$ & $\begin{array}{l}28 \text { indivíduos, ambos os sexos, } \\
\text { idade entre } 30 \text { e } 60 \text { e não } \\
\text { diabéticos. }\end{array}$ & $\begin{array}{l}\text { Redução da concentração sérica de glicose e } \\
\text { melhora no perfil lipídico no grupo da farinha da } \\
\text { casca de maracujá. }\end{array}$ \\
\hline Queiroz et al. (2012) & $\begin{array}{l}30 \mathrm{~g} / \text { dia de farinha de maracujá } \\
60 \text { dias. }\end{array}$ & $\begin{array}{l}43 \text { pacientes com DM2, ambos os } \\
\text { sexos, com idade entre } 57 \text { e } 73 \\
\text { anos. }\end{array}$ & Redução da glicemia e hemoglobina glicada. \\
\hline $\begin{array}{l}\text { Alexandrino et al. } \\
(2012)\end{array}$ & $\begin{array}{l}30 \mathrm{~g} / \mathrm{dia} \text { via oral; } \\
60 \text { dias. }\end{array}$ & $\begin{array}{l}33 \text { pacientes, ambos os sexos, com } \\
\text { DM2, com idade entre } 40 \text { e } 80 \\
\text { anos. }\end{array}$ & $\begin{array}{l}\text { Não houve redução da glicemia e hemoglobina } \\
\text { glicada em DM } 2 \text {. }\end{array}$ \\
\hline Miranda et al. (2011) & $\begin{array}{l}30 \mathrm{~g} \text { de farinha de maracujá ao dia; } \\
60 \text { dias. }\end{array}$ & $\begin{array}{l}16 \text { pessoas não diabéticas com } \\
\text { idade entre } 30 \text { e } 60 \text { anos. }\end{array}$ & $\begin{array}{l}\text { Houve diminuição significativa das } \\
\text { concentrações de glicose e de colesterol total. }\end{array}$ \\
\hline
\end{tabular}

Fonte: As Autoras.

Há maior quantidade de fibras na casca do maracujá amarelo que na polpa deste fruto, além disso, outras substâncias também podem ser observadas, como os compostos fenólicos, que proporcionam a função antioxidante a esta fruta e, por esta razão, a farinha da casca de maracujá amarelo pode ser considerada um complemento alimentar (Claro, Rodrigues \& Teixeira, 2018). A inclusão de alimentos ricos em fibras, tais como cereais, legumes, frutas e suplementos de fibra dietética, tem demonstrado grande potencial na redução dos níveis de glicose no plasma sanguíneo e nos níveis de hemoglobina glicada 
(HbA1c), o que associa a melhora do controle glicêmico de indivíduos com DM2 ao aumento do consumo de fibras (Silva et al., 2018). Com isso, diversos estudos têm sido realizados com o intuito de verificar os efeitos do consumo da fibra de maracujá envolvendo esta patologia.

Diante disso, a maioria dos estudos realizados com humanos analisados neste trabalho demonstraram a eficácia da suplementação de 30g/dia da farinha da casca de maracujá na redução da glicemia de jejum (GJ) e na HbA1c de indivíduos com DM2, exceto o estudo realizado por Gaspar, Araújo \& Uchoa (2016), que utilizaram $12 \mathrm{~g} / 3$ vezes ao dia) de farinha de maracujá adicionada a algum alimento e não demonstrou significância estatística para a redução da HbA1c. O estudo realizado por Castro (2019) com 56 indivíduos com diagnóstico de DM2, verificou a eficácia da ingestão de 3 cápsulas de 500mg por dia antes das principais refeições (desjejum, almoço e jantar), consumindo dessa maneira, o equivalente a 4,5g da farinha durante 120 dias (4 meses). Com isso, o autor ao final do estudo, constatou que a GJ e a HbA1c apresentaram melhoras estatisticamente significantes. Em relação ao controle lipídico, notou-se melhora em algumas variáveis, como LDL-C e HDLC, porém, sem significância estatística, o que pode-se concluir que a farinha de maracujá não demonstrou a mesma efetividade para o perfil lipídico, o que pode estar relacionado com a quantidade de farinha da casca de maracujá consumida.

Além disso, no estudo realizado por Queiroz et al. (2012) avaliou-se o efeito da Passiflora edulis flavicarpa Deg. na sensibilidade insulínica de pacientes com DM2, evidenciando o aumento nos níveis de insulina nos homens avaliados. Ademais, em algumas pesquisas, os indivíduos participantes relataram desconforto gastrointestinal, sonolência, dor gástrica, forte sabor residual e plenitude gástrica (Cunha, 2018; Miranda et al., 2013; Queiroz et al., 2012), o que pode interferir na aderência ao tratamento (Cunha, 2018), que foi o caso do estudo realizado por Araújo et al. (2017), que mesmo verificando o efeito da farinha da casca de maracujá amarelo no controle da glicemia em indivíduos com DM2, os autores alertaram para as limitações da realização do estudo devido a adesão ao consumo da farinha pelos motivos exemplificados anteriormente.

Quanto ao sabor residual relatado em alguns estudos analisados, Li \& Schluesener (2017) afirmam que o sabor residual forte encontrado na farinha do maracujá amarelo, é proveniente de uma substância presente no mesocarpo do maracujá denominada hesperidina, um flavonoide que pode ser encontrado com abundância no endocarpo, mesocarpo e exocarpo de frutas cítricas e que pode ser fermentado pela microbiota intestinal, passando para a forma aglicona, que é mais facilmente absorvida.

Com o intuito de cooperar com os estudos supracitados que foram desenvolvidos com humanos, alguns estudos realizados com ratos foram selecionados a fim de fazer um comparativo. De Faveri et al. (2020) em seu estudo com ratos, avaliaram os efeitos da farinha da casca de maracujá nos distúrbios metabólicos causados pela dieta de cafeteria, que simula os hábitos alimentares humanos, recendo esse nome devido o consumo de alimentos hipercalóricos, ricos em carboidratos e lipídios, como chocolate ao leite, amendoim e biscoito doce. Os autores verificaram um efeito positivo do consumo da farinha da casca de maracujá, pois os resultados apontaram a redução do ganho de peso corporal, a deposição de gordura, predominantemente no fígado, e melhora em relação a tolerância à glicose e a sensibilidade à insulina nas alterações metabólicas causadas pela dieta da cafeteria.

Um estudo similiar foi realizado por Goss et al. (2018), teve como objetivo investigar os efeitos da suplementação da farinha da casca de maracujá sobre a sensibilidade à insulina, adiposidade e parâmetros metabólicos em ratos jovens que receberam bebidas enriquecidas com 10\% de frutose por 8 semanas. A ingestão de frutose induziu resistência à insulina, aumento dos níveis de triglicerídeos séricos, crescimento de depósitos de gordura no fígado e alargamento do diâmetro dos adipócitos. Em contrapartida, o grupo que recebeu PEPF não apresentou tais anormalidades, que podem estar relacionadas à presença de fibras insolúveis ou compostos bioativos presentes na sua composição. Assim, os autores demonstraram que a suplementação com a farinha da casca de maracujá amarelo previne a resistência à insulina e a esteatose hepática induzida por dieta pobre em frutose em ratos jovens. 
Além da eficácia na redução da glicemia, um estudo realizado por Figueiredo et al. (2016), avaliou se o consumo de farinha de maracujá provocaria ganho de peso corporal em ratos Wistar. Os autores verificaram que o grupo de ratos que consumiram tanto farinha da casca de maracujá integralmente quanto a elaborada em associação do albedo e flavedo apresentaram menor ganho de peso corporal A ingestão da farinha da casca do maracujá por possuir fibras solúveis, como a pectina, promove maior consumo de fibras, que pode ser associada à perda de peso ou menor ganho de peso, devido a sua capacidade de diminuir o esvaziamento gástrico, além de influenciar a oxidação e armazenamento de gordura. De acordo com Lima et al. (2016) também podem melhorar a sensibilidade à insulina e modular os hormônios como polipeptídio intestinal (PYY) e o peptídeo-1, semelhante ao glucagon (GLP-1), que vão agir na secreção da insulina e no controle da ingestão de alimentos.

Apesar dos efeitos positivos demonstrados nos resultados dos estudos, deve-se considerar as diferenças nas metodologias, questão das doses diferenciadas entre os artigos, a diferença na faixa etária, o tipo de dieta, a realização de atividade física que podem contribuir para os efeitos na redução da glicemia e que por isso não podemos atribuir a melhora da glicemia somente ao consumo de farinha de maracujá.

Portanto, sendo assim, é importante salientar que pacientes diabéticos que queiram aumentar o consumo de fibras na alimentação devem procurar a ajuda de um profissional nutricionista, a fim de que este possa reorientá-los em relação ao uso de insulina e/ou hipoglicemiantes orais com o intuito de evitar a hipoglicemia (baixa concentração sanguínea de glicose), uma vez que a utilização de fibras tende a resultar no aumento do tempo de absorção e na redução da quantidade de carboidratos absorvidos (Moreira, 2011).

\section{Conclusão}

Após as pesquisas e resultados obtidos, pode-se concluir que a farinha da casca de maracujá apresenta resultados positivos quanto à diminuição da glicemia e hemoglobina glicada, relacionada diretamente com a pectina presente em sua casca, cuja atividade gelificante proporciona a capacidade de diminuir a velocidade de absorção dos carboidratos e lipídios. Além disso, os estudos apontaram efeitos benéficos no perfil lipídico, triglicerídeos, redução dos níveis pressóricos e peso.

Deste modo, a utilização da suplementação de $30 \mathrm{~g}$ (equivalente a 3 colheres de sopa) da farinha da casca do maracujá amarelo durante 60 dias, administrados via oral por meio de alimentos tais como sucos, leite, vitaminas ou mesmo misturado às refeições, serviu como alimento enriquecedor das dietas de indivíduos com DM2, podendo gerar benefícios à saúde.

Recomenda-se que mais estudos experimentais e clínicos sejam desenvolvidos para avaliar o potencial de toxicidade da farinha do maracujá amarelo em diferentes dosagens, formas de administração e período de tratamento, para que assim, possa ser estabelecida uma quantidade que represente o limite de segurança para consumo alimentar sem ocorrência de efeitos indesejáveis à saúde.

\section{Referências}

Alexandrino, L. S. B., Rumiato, A. C., Alexandrino, A. P \& Lavado, E. L. (2012). O efeito da farinha do maracujá amarelo no controle do diabetes mellitus tipo 2. Anais $6^{\circ}$ Congresso Nacional de Extensão Universitária. $15^{\circ}$ Encontro de Atividades Científicas da Unopar. https://repositorio.pgsskroton.com/bitstream/123456789/2693/1/O\%20uso\%20da\%20farinha\%20do\%20maracuj\%C3\%A1.pdf.

Alvarenga, C. F., Lima, K. M. N., Mollica, L. R., Azeredo. L. O. \& Carvalho, C. (2017). Uso de plantas medicinais para o tratamento do diabetes mellitus no Vale do Paraíba-SP. Revista de Ciência da Saúde, 2(2), 36-44. https://revistaeletronicafunvic.org/index.php/c14ffd10/article/view/84/73.

Ambrósio-Ugri, M. C. B. \& Ramos, A. C. H. (2012). Elaboração de barra de cereais com substituição parcial de aveia por farinha da casca de maracujá. Revista Tecnológica, 21(1), 69-76. doi: 10.4025/revtecnol.v21i1.10611.

Brasil. Ministério da Saúde. Plataforma IVIS - Plataforma Integrada de Vigilância em Saúde (2021). Painel de Monitoramento COVID-19. http://plataforma.saude.gov.br/coronavirus/covid-19/. 
Brasil. Ministério da Saúde. Secretaria de Atenção à Saúde. Departamento de Atenção Básica. (2013). Estratégias para o cuidado das pessoas com doença crônica: diabetes $\quad$ mellitus. Caderno $\quad$ de $\quad$ Atenção https://bvsms.saude.gov.br/bvs/publicacoes/estrategias_cuidado_pessoa_diabetes_mellitus_cab36.pdf

Brasil. Ministério da Saúde. Secretaria de Vigilância em Saúde. (2019). Vigilância de fatores de risco e proteção para doenças crônicas por inquérito telefônico: estimativas sobre frequência e distribuição sociodemográfica de fatores de risco e proteção para doenças crônicas nas capitais dos 26 estados brasileiros e no Distrito Federal em 2019. https://bvsms.saude.gov.br/bvs/publicacoes/vigitel_brasil_2019_vigilancia_fatores_risco.pdf.

Bruning, M. C. R., Mosegui, G. B. G. \& Vianna, C. M. M. (2012). A utilização da fitoterapia e de plantas medicinais em unidades básicas de saúde nos municípios de Cascavel e Foz do Iguaçu - Paraná: a visão dos profissionais de saúde. Ciência \& Saúde Coletiva, 17 (10), 2675 - 2685. doi:10.1590/S141381232012001000017

Cádiz-Gurrea, M. L., Villegas-Aguilar, M., Leyva-Jiménez, F. J., Pimentel-Moral, S., Fernández-Ochoa, Á., Alañón, M. E. \& Segura-Carretero, A. (2020). Revalorization of bioactive compounds from tropical fruit by-products and industrial applications by means of sustainable approaches. Food research international, 138(Pt B), 109786. Doi:10.1016/j.foodres.2020.109786

Canteri, M. H., Wosiacki, L. M. G. \& Scheer, A. P. (2012). Pectina: da matéria-prima ao produto final. Polímeros,22 (2), 149-157, 2012. doi: 10.1590/S010414282012005000024

Cazarin, C. B. B., Silva, J. K., Colomeu, T. C., Zollner, R. de L. \& Junior, M. R. M. (2014). Capacidade antioxidante e composição química da casca de maracujá (Passiflora edulis). Ciência Rural, 44 (9). doi: 10.1590/0103-8478cr20131437.

Claro, M. L., Rodrigues, G. P. \& Teixeira, S. A. (2018). Propriedades funcionais da casca do maracujá amarelo (Passiflora edulis) na síndrome metabólica. Demetra, 13 (1), 181 - 194. doi: 10.12957/demetra.2018.28957.

Costa, B. F., Liz, F. R., Ferreira, J. G. S., Santos, R. C., Gonçalves, T. H. \& Balbi. B. E. (2018). Uso da farinha da casca do maracujá amarelo (Passiflora edulis f. flavicarpa deg. - Família Passifloracea) na formulação do macarrão caseiro. Visão Acadêmica, 19 (4). doi:10.5380/acd.v19i4.65496.

Costa, E. L. (2015). Produção e aceitação de massa fresca tipo talharim enriquecida com farinha de maracujá (Passiflora edulis f. var. flavicarpa) e verificação do seu efeito na glicemia. Nutrire. 40 (3), 352-360. doi:10.4322/2316-7874.71315.

Cunha, M. C. S. O. (2018). Efeito da farinha do albedo do maracujá amarelo no controle glicêmico de pessoas com diabetes tipo 2 na atenção primária à saúde. Dissertação de Mestrado em Enfermagem, Universidade da Integração Internacional da Lusofonia Afro-Brasileira., Redenção, Ceará, Brasil. https://repositorio.unilab.edu.br/jspui/bitstream/123456789/753/1/DISSERTAC\%CC\%A7A\%CC\%83O\%20-\%20\%20FINAL\%2026.02.pdf.

Cunha, M. R., Campos, L. F. \& Silva, J. G. N. (2017). Diabetes Mellitus tipo 2. In: Waitzberg, D. L. Nutrição Oral, enteral e Parenteral na Prática Clínica. 5 ed. Rio de Janeiro: Atheneu.

de Araújo, M., Veras, V. S., Freitas, R., Paula, M., Araújo, T. M., Uchôa, L., Gaspar, M., Cunha, M., Serra, M., Carvalho, C., Costa, E. C. \& Damasceno, M. (2017). Efeito da farinha da casca do maracujá-amarelo no controle glicêmico de pessoas com diabetes mellitus tipo 2 : ensaio clínico randomizado. Journal of diabetes and metabolic disorder, 16, 18. doi:10.1186/s40200-017-0300-z.

De Faveri, A., De Faveri, R., Broering, M. F., Bousfield, I. T., Goss, M. J., Muller, S. P., Pereira, R. O., Silva, A. M. O., Machado, I. D., Quintão, N. L. M. \& Santin, J. R. (2020). Effects of passion fruit peel flour (Passiflora edulis f. flavicarpa O. deg.) in cafeteria diet-indiced metabolic disorders. Journal of Ethnopharmacology, v. 250. doi:10.1016/j.jep.2019.112482.

Dias, J. F., Vianna, L. M., Souza, A. N. R., Miranda, A. F., Balthar, R. O. \& Ciniglia, N. Nutrição, Doenças Cronico-Degenerativas e COVID-19. (2020). Ebook, 1 ed, 7 - 10. Rio de Janeiro: UNIRIO. Disponível em: http://www.unirio.br/covid/material-educativo/COVID19NUTRICAO1507.pdf.

Dias, P. C., Henriques, P., Dos Anjos, L. A. \& Burlandy, L. (2017). Obesidade e políticas públicas: concepções e estratégias adotadas pelo governo brasileiro. Cadernos de Saúde Pública, 33 (7). doi:10.1590/0102-311x00006016.

Dias da Silva, A., Matos Júnior, N., Damasceno, D. D., Guimarães, N. S. \& Gomes, J. M. G. (2020). Estado nutricional, fatores de risco e comorbidades em adultos portadores de diabetes mellitus tipo 2. HU Revista, 46, 1-9. doi:10.34019/1982-8047. 2020.v46.28790.

Elias, C. S. et al. (2012). Quando chega o fim? Uma revisão narrativa sobre terminalidade do período escolar para alunos deficientes mentais. SMAD: Revista Electrónica en Salud Mental, Alcohol y Drogas, 8 (1), 48-53. http://pepsic.bvsalud.org/pdf/smad/v8n1/08.pdf.

Ferreira, M. F. P. \& Pena, R. S. (2010). Estudo da secagem da casca do maracujá amarelo. Revista Brasileira de Produtos Agroindustriais, 12 (1),15 - 28. doi: 10.15871/1517-8595/rbpa. v12n1p15-28.

Figueiredo, D. A. F., Pordeus, L. C. M., Paulo, L. L., Braga, R. M., Fonsêca, D. V., Sousa, B. S., Costa, M. J. C., Gonçalves, M. C. R. \& Oliveira, K. H. D. (2016). Effects of bark flour of Passiflora edulis on food intake, body weight and behavioral response of rats. Revista Brasileira de Farmacognosia, 26(5), 595 - 600. doi:10.1016/j.bjp.2016.02.010.

Forcina, D. V., Almeida, B. O. \& Ribeiro-Jr, M. A. F. (2008). Papel da cirurgia bariátrica no controle do diabete melito tipo II. Arquivos Brasileiros de Cirurgia Digestiva, 21 (3), 130 - 132. https://www.scielo.br/j/abcd/a/yLtV3GZbptVK8SgsFbwXtKP/?lang=pt\&format=pdf.

Franz, M. J., \& Evert, A. B. (2018). Terapia de Nutrição Médica para Diabetes Mellitus e hipoglicemia de origem não diabética. Mahan, L. K., Escott-Stump, S. \& Raymond, J. L. Krause: Alimentos, Nutrição e Dietoterapia. 14 ed. Rio de Janeiro: Elsevier.

Gaspar, M. W. G., Araújo, M. F. M., Uchoa, L. R. A. (2016). Efeito da farinha do albedo de maracujá amarelo no controle glicêmico de pessoas com diabetes. Anais da III Semana Universitária: Ética na formação acadêmica. https://docplayer.com.br/72820274-Efeito-da-farinha-do-albedo-do-maracuja-amarelo-nocotrole-glicemico-de-pessoas-com-diabetes.html. 
Goss, M. J., Nunes, M. L. O., Machado, I. D., Merlin, L., Macedo, N. B., Silva, A. M. O., Bresolin, T. M. B. \& Santin, J. R. (2018). Peel flour of Passiflora edulis Var. Flavicarpa supplementation prevents the insulin resistance and hepatic steatosis induced by low-fructose-diet in young rats. Biomedicine \& Pharmacotherapy, 102, 848 -854. doi: 10.1016/j.biopha.2018.03.137.

IDF. International Diabetes Federation (2019). IDF Atlas, 9th edn. Brussels, Belgium. http://www.diabetesatlas.org.

Kahn, S. E., Cooper, M. E. \& Del Plato, S. (2014). Pathophysiology, and treatment of type 2 diabetes: perspectives on the past, present, and future. The Lancet, 383 (9922),1068-1083. doi:10.1016/S0140-6736(13)62154-6.

Lattimer, J. M., Haub, M. D. (2010). Effects of Dietary Fiber and Its Components on Metabolic Health. Nutrients, 2, 1266-1289. doi:10.3390/nu2121266. Li, C. \& Schluesener, H. (2017). Health - promoting effects of the citrus flavone hesperidin. Critical reviews in food science and nutrition, 57 (3), $613-631$. doi:10.1080/10408398.2014.906382.

Lima, G. C., Vuolo, M. M., Batista, Â. G., Dragano, N. R., Solon, C., \& Maróstica Junior, M. R. (2016). Passiflora edulis peel intake improves insulin sensitivity, increasing incretins and hypothalamic satietogenic neuropeptide in rats on a high-fat diet. Nutrition, 32 (7-8), 863-870. doi:10.1016/j.nut.2016.01.014

Lupatini, A. L., Fudo, R. M., Mesomo, R. C., Conceição, W. A. S. \& Coutinho, M. R. (2011). Desenvolvimento de biscoitos com farinha de casca de maracujá e okara. Revista Ciências Exatas e Naturais, 13 (3), 317 - 329. https://revistas.unicentro.br/index.php/RECEN/article/view/1355/1550.

Miranda, A. A., Caixeta, A. C. A., Flávio, E. F. \& Pinho, L. (2013). Desenvolvimento e análise de bolos enriquecidos com farinha da casca do maracujá (Passiflora edulis) como fonte de fibras. Alimentos e Nutrição Araraquara, $24 \quad(2), \quad 225 \quad-232 . \quad$ http://servbib.fcfar.unesp.br/seer/index.php/alimentos/article/view/225/2173.

Miranda, G. S., Rennó, L. N., Machado, B. B., Silva, J. L., Pinto, R. \& Oliveira, M. R. (2011). Efeito da farinha da casca de maracujá sobre os níveis séricos

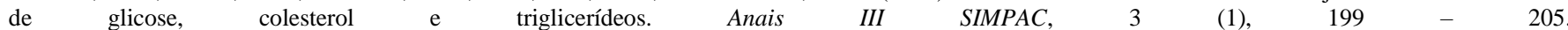
https://academico.univicosa.com.br/revista/index.php/RevistaSimpac/article/view/353/516.

Miranda, G. S., Rennó, R. N., Machado, B. B., Silva, J. L., Pinto, R. \& Oliveira, M. R. (2014). Efeito do consumo da aveia e farinha da casca de maracujá sobre a glicemia e lipemia em um grupo de voluntários. Revista de Ciências Farmacêuticas Básica e Aplicada, 35 (2), $245-250$. https://academico.univicosa.com.br/revista/index.php/RevistaSimpac/article/view/353.

Moreira, F. D. (2011). Resposta glicêmica aguda e saciedade após adição alternada de farelo de trigo, farinha de maracujá e pó de algas marinhas no desjejum de diabéticos tipo 2. Dissertação de Mestrado em Ciência da Saúde, Universidade Federal de Brasília, Distrito Federal, Brasília, Brasil. https://repositorio.unb.br/bitstream/10482/10090/1/2011_FernandaDuarteMoreira.pdf.

Muñoz-Almagro, N., Montilla, A. \& Villamiel, M. (2021). Role of pectin in the current trends towards low-glycaemic food consumption. Food Research International, 140. doi: 10.1016/j.foodres.2020.109851.

Navolar, T. S., Tesser, C. D. \& Azevedo, E. (2012). Contribuições para a construção da Nutrição Complementar Integrada. Interface - Comunicação, Saúde, Educação, 16 (41). doi:10.1590/S1414-32832012005000024.

Oliveira, C. F., Gurak, P. D., Cladera-Olivera, F. \& Marczak, L. D. F. (2016). Evaluation of physicochemical, technological and morphological characteristics of powdered yellow passion fruit peel. International Food Research Journal, 23 (4), 1653 - 1662. http://www.ifrj.upm.edu.my/23\%20(04)\%202016/(40).pdf. Oliveira, D. K. B. (2016). Aplicação e aceitabilidade da farinha da casca do maracujá amarelo (Passiflora edulis) na alimentação de um grupo de idosos. Dissertação de Mestrado em Gerontologia, Universidade Católica de Brasília. Brasília, Brasil. https://bdtd.ucb.br:8443/jspui/handle/tede/2174.

Orioli, L., Hermans, M. P., Thissen, J. P., Maiter, D., Vandeleene, B. \& Yombi, J. C. (2020). COVID-19 in diabetic patients: Related risks and specifics of management. Annales d'endocrinologie, 81(2-3), 101-109. doi:10.1016/j.ando.2020.05.001

Ozores, B, Storck, C. R. \& Fogaça, A. O. (2015). Aceitabilidade e características tecnológicas de bolo enriquecido com farinha de maracujá. Revista Disciplinarum Scientia. 16 (1), 61-69. https://periodicos.ufn.edu.br/index.php/disciplinarumS/article/view/1110/1052.

Petermann, F., Díaz-Martínez, X., Garrido-Méndez, A., Leiva, A. M., Martínez, M. A., Salas, C., Poblete-Valderrama, F. \& Celis-Morales, C. (2018). Asociación entre diabetes mellitus tipo 2 y actividade física em personas com antecedentes familiares de diabtes. Gaceta sanitária, 32 (3), 230 - 235. doi:10.1016/j.gaceta.2017.09.008

Rojas-Padilla, I., Zambrano-Ríos, D. \& Matta-Miramar, A. (2020). Evaluación de la influencia del estado nutricional en el control de diabetes mellitus tipo 2. Duazary, 17 (2), 10 - 19. doi:10.21676/2389783X.3232.

Queiroz, M. S. R., Janebro, D. I., Cunha, M. A. L., Medeiros, J. dos S., Sabaa-Srur, A. U. O., Diniz, M. de F. F. M. \& Santos, S. c dos. (2012). Effect of the yellow passion fruit peel flour (Passiflora edulis f. flavicarpa deg.) in insulin sensitivity in type 2 Diabetes Mellitus patients. Nutrition Journal, 11 (89). doi:10.1186/1475-2891-11-89.

Santana, F. C., Silva, J. V., Santos, A. J. A. O., Alves, A. R., Wartha, E. R. S. A., Marcellini, O. S. \& Silva, M. A. A. P. (2011). Desenvolvimento de biscoito rico em fibras elaborado por substiruição parcial da farinha de trigo por farinha da casca do maracujá amarelo (Passiflora edulis flavicarpa) e fécula de mandioca (Msnihot esculenta crantz). Revista Alimentos e Nutrição Araraquara, $22 \quad(3), \quad 391 \quad-\quad 399 . \quad$ http://servbib.fcfar.unesp.br/seer/index.php/alimentos/article/view/1646/1646.

Santos, F. G. N. (2017). História, uso local e caracterização física e química do maracujá-amarelo (Passiflora edulis fo. flavicarpa O. Deg.). Monografia, Universidade Federal de Campina Grande. http://serv-bib.fcfar.unesp.br/seer/index.php/alimentos/article/view/1646/1646.

SBD. Sociedade Brasileira de Diabetes (2017). Diretrizes da Sociedade Brasileira de Diabetes 2017 - 2018. Epidemiologia e impacto global do diabetes mellitus, p. 12 - 19. https://www.diabetes.org.br/profissionais/images/2017/diretrizes/diretrizes-sbd-2017-2018.pdf.

SBD. Sociedade Brasileira De Diabetes. (2019). Diretrizes da Sociedade Brasileira de Diabetes 2019 - 2020. Tratamento do diabetes mellitus: medidas de estilo de vida, p. 96 - 163. Disponível em: https://www.diabetes.org.br/profissionais/images/DIRETRIZES-COMPLETA-2019-2020.pdf. 
Research, Society and Development, v. 10, n. 9, e54910918404, 2021

(CC BY 4.0) | ISSN 2525-3409 | DOI: http://dx.doi.org/10.33448/rsd-v10i9.18404

Silva, M. J. R. (2018). Avaliação clínica e bioquímica de pacientes diabéticos em hemodiálise suplementados com a farinha da casca do maracujá amarelo. Trabalho de Conclusão de Curso, Universidade Federal de Campina Grande. Cuité, Paraíba. http://dspace.sti.ufcg.edu.br:8080/jspui/bitstream/riufcg/6954/1/MIKAEL\%20JOHNATHAN\%20RIBEIRO\%20DA\%20SILVA\%20-

\%20TCC\%20NUTRI\%c3\%87\%c3\%830\%202018.pdf.

Talia, C., Connolly, L. \& Fowler, P. A. (2021). The insulin-like growth factor system: A target for endocrine disruptors? Environment International, 147. doi:10.1016/j.envint.2020.106311.

Vuolo, M. M., Lima, G. C., Batista, Â. G., Carazin, C., Cintra, D. E., Prado, M. A. \& Júnior, M. (2020). Passion fruit peel intake decreases inflammatory response and reverts lipid peroxidation and adiposity in diet-induced obese rats. Nutrition Research, 76, 106-117. doi: 10.1016/j.nutres.2019.08.007.

Zanaib Shahid, B. S. (2020). COVID-19 and Older Adults: What We Know. Journal of the American Geriatrics Society, 68 (5), 926 - 929. doi:10.1111/jgs.16472.

Zhang, Y., Cui, Y., Shen, M., Zhang, J., Liu, B., Dai, M., Chen, L., Han, D., Fan, Y., Zeng, Y., Li, W., Lin, F., Li, S., Chen, X., \& Pan, P. (2020). Medical team from Xiangya Hospital to support Hubei, China. Association of diabetes mellitus with disease severity and prognosis in COVID-19: A retrospective cohort study. Diabetes research and clinical practice, 165, 108227. doi: 10.1016/j.diabres.2020.108227.

Zhu, L., She, Z. G., Cheng, X., Qin, J. J., Zhang, X. J., Cai, J., Lei, F., Wang, H., Xie, J., Wang, W., Li, H., Zhang, P., Song, X., Chen, X., Xiang, M., Zhang, C., Bai, L., Xiang, D., Chen, M. M., Liu, Y., ... Li, H. (2020). Association of Blood Glucose Control and Outcomes in Patients with COVID-19 and Preexisting Type 2 Diabetes. Cell metabolism, 31(6), 1068-1077.e3. doi:10.1016/j.cmet.2020.04.021. 